\title{
Communication Innovation and Text Messaging Based Lingual Creativity
}

\author{
Asst. Prof. Ali Muhsen Gharab Al-Majdawi \\ Wasit University, College of Education for Human Sciences
}

DOI: $10.37648 / \mathrm{ijrssh.v10i03.045}$

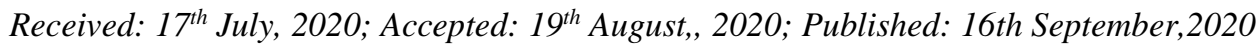

\begin{abstract}
Language is changed as anything in our life that is changing. Such a change can be either syntactically, semantically, or phonologically. That change can be utilized in developing the way of communication, and effect even the number of letters that can be used in a word or a sentence. The study aims at identifying Communication Innovation and Text Messaging based Lingual Creativity. The subjects of this study are a group of forty-two students were they are pre and post graduate levels of different majors. The subjects are mixed gender of male and female. Age are considered and separated in five sections. The subjects are chosen according to their desire to participate in the questionnaire and their familiarity with the statements. In order to get as accurate results as possible, the questionnaire has been used as an instrument the result show that the first item of the questionnaire Does computer communication contribute to meet the requirements of the digital era? has got high degree which is $95.3 \%$ and mean which is 78.571
\end{abstract}

Keywords: Communication Innovation ,Text Messaging , Lingual Creativity

\section{INTRODUCTION}

\subsection{The Problem and its Significant:}

The language used in texting is somewhat more proper and closely related to the traditional written language. It has the qualities of being complete and clear which are essential in getting a message delivered clearly. Texting can be incredibly simple. "You can fill your role of returning calls and keep in touch with people without any pressure to be creative or witty. We're talking about language in its most stripped down kind of level.

The use of non-standard forms online will put the future of Standard English in danger. They add that they are driven to use short forms which are used because of "the urgency of turn-taking and the ease of typing and the urge to save time and space". Moreover, since they already know standard spellings of words prior to abbreviating them, it is not a matter to be concerned with, whether it will affect their academic writing or not. In addition, concerning the language used on character- 5 limited, Twitter users are given the opportunity to play around with words to get them to fit into the constraint and it is really fun to do besides encouraging the users to be efficient in constructing a tweet Knapp, 2011,(cited in Nabila, 2014: 34). In this case, ,language playe means users tend to manipulate linguistic forms in the act of communicating Jones, 2010 (cited in Nabila, 2014: 34). However, it is not always about form. creativity in the new media also involves wordplay (e.g. punning), type play (e.g. repetition) and sound play (e.g. accent stylization, letternumber homophones) (Thurlow, 2011:7).. 
Yet, creativity in utterances or writing may not only lie in the ,language play" rhetorical devices. According to Jones (2010) (cited in R. Rubdy and L. Alsagoff, 2013:172), "What may be „creative" may have more to do with the strategic way language is used, and what may be „,reated may not be an inventive linguistic product, but rather a new way of dealing with a situation or a new set of social relationships." He further explains that lingual creativity exist when writers code-mix and shift styles in their texts based on their social and cultural contexts. When it involves more than one language, this is termed as „bilingual creativity"e.

\subsection{Question of the Research:}

The question of this study about what is the relation between Communication Innovation and Text Messaging based Lingual Creativity

\subsection{The Aim of the Study :}

The study aims at identifying Communication Innovation and Text Messaging based Lingual Creativity

\section{LITERATURE REVIEW}

\subsection{Lingual Creativity and Communication Innovation}

According to Tagliamonte and Denis,(2008:12) in their findings, the use of shortened words and abbreviations is relatively small in Instant messaging; it does not appear that extensively as for how the media puts it. Instead, these features demonstrate the creativity of users to construct their messages, and most of the time what they want to deliver is successfully conveyed despite the non-standard forms of language that they use. Hence, rather than seeing it as degrading a language, it should be seen as users having an innovative and a good command of the language.

Another study by. Aziz et al, (2013:12886) have contradicted the belief that the use of non-standard forms online will put the future of Standard English in danger. They add that they are driven to use short forms which are used because of "the urgency of turn-taking and the ease of typing and the urge to save time and space". Moreover, since they already know standard spellings of words prior to abbreviating them, it is not a matter to be concerned with, whether it will affect their academic writing or not. In addition, concerning the language used on character- 5 limited, Twitter users are given the opportunity to play around with words to get them to fit into the constraint and it is really fun to do besides encouraging the users to be efficient in constructing a tweet Knapp, 2011,(cited in Nabila, 2014: 34). In this case, „language play" means users tend to manipulate linguistic forms in the act of communicating Jones, 2010 (cited in Nabila, 2014: 34). However, it is not always about form. creativity in the new media also involves wordplay (e.g. punning), type play (e.g. repetition) and sound play (e.g. accent stylization, letternumber homophones, Thurlow, 2011:7).

Yet, creativity in utterances or writing may not only lie in the „language play", puns, metaphors or other rhetorical devices. According to Jones (2010) (cited in R. Rubdy and L. Alsagoff, 2013:172), "What may be „creative"e may have more to do with the strategic way language is used, and what may be ,created "e may not be an inventive linguistic product, but rather a new way of dealing with a situation or a new set of social relationships." He further explains that lingual creativity exist when writers code-mix and shift styles in their texts based on their social and cultural contexts. When it involves more than one language, this is termed as „bilingual creativity“.

\subsection{Texting}

Texting is a slang term that refers to the creation and transmission of short electronic text messages between two or more mobile device users over a network. This usually means sending messages with no more than 100 characters, although it has now been extended to include audio, images and video content, known as multimedia message service (MMS). The term texting was originally used as a shorter form of short message service (SMS), which allowed only 144 pure text characters per message

Texting refers to the sending of short typed messages between mobile phones using the Short Message Service (SMS), a feature of most mobile phones since the late 1990s. In much of the European literature into texting, the term SMS is also used to describe both the medium and the messages. Kasesniemi and Rautianen,(2002:180). Short text messages system is a powerful medium of electronic communication, which is a fast, cheap and convenient way of transferring information from one 
mobile device to other in the range of 160 characteristics. Bertel et al,2012,(cited in Topolska, 2015:18).

Texting reflects language change and innovation in language. Variation within text message depends on the particular use of SMS. (Ong ${ }^{\text {ee }}$ onda,2009:3).

The dominant features in SMS language are the use of abbreviations, slang, syntactic reductions, asterisk emoting, emoticons, deletions of parts of speech, especially subject pronoun, preposition, articles, copula, auxiliary or modal verbs and contractions. The unique use of SMS language serves to tie the group together through the development of a common history (Ling,2000:18).

a) the comparatively short length of text messages;

b) the relative concentration of non-standard typographic markers; and
Their predominantly small-talk content and solidary orientation. Key qualifications here are „combination ${ }^{\text {ee }}$, „,comparatively ${ }^{\text {ee }}$,,relative ${ }^{\text {ee }}$ and ,,predominantlye; none of these generic and stylistic features is sufficient individually to characterize texting.

\subsection{The Use of Emoticons}

In face-to-face interactions, nonverbal behaviour communicates quite a lot about intent. Those behaviors such as facial expressions, the placement of head and shoulders, the use of hands can deliver information, regulate the interaction, and express feelings and intimacy. In online communication, emoticons may be used to help achieve the same thing by serving as"nonverbal surrogates". (Derks, Bos, and Grumbkow, 2008: 843).

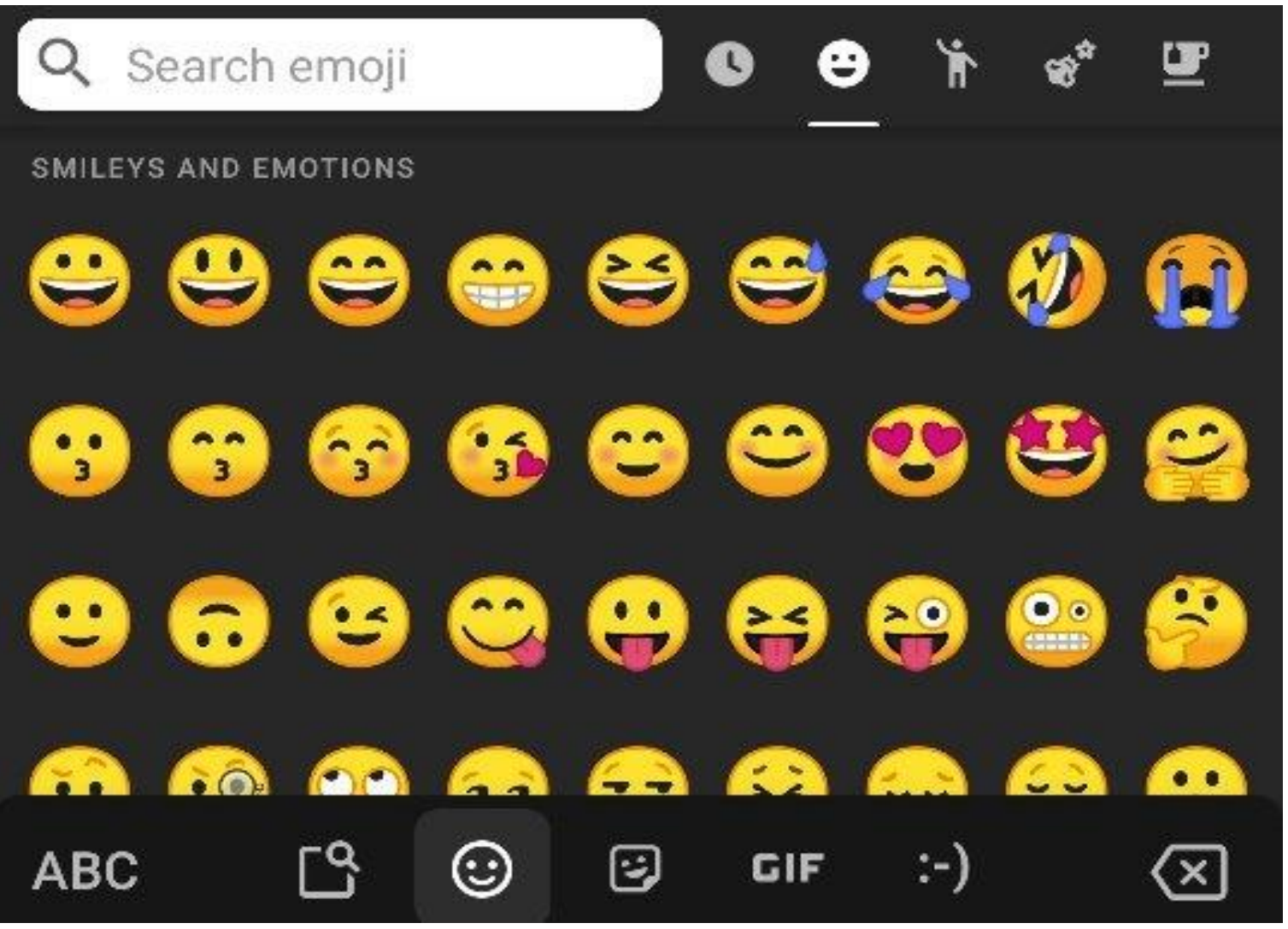

Emoticons are "graphic representations of facial expressions". Walther and D"Addario,( 2001: 324), Which deliver emotional rather than task-oriented information . and index a user"s effective stance. Most emoticons are well known and 
commonly recognized symbols among users of EMC. They often act as substitutes or surrogates for nonverbal cues, which are usually absent in text-based EMC. Sometimes they are used as a compliment to a text message. Smiling is a common human reaction mostly used to indicate happiness, hence it is not surprising that it has found a symbolic representation in EMC in the form of emoticons and smileys. There are two types of smileys, the icon (or emoji), which pictorially represents a smiling human face and keystroke-based symbolic emoticons such as :-).

They have the same impact in terms of how a message is interpreted. However, some argue that the emoji smiley has a stronger impact on the personal mood of the viewer than a keystroke-based emoticon this may be due to the wide range of emoji icons now possible in EMC, or because emoji is a more realistic portrayal of human expressions.

\section{PROCEDURES}

\section{.3.1The Subjects}

The subjects of this paper are a group of forty-two students were they are pre and post graduate levels of different majors. The subjects are mixed gender of male and female. Age are considered and separated in five sections. The subjects are chosen according to their desire to participate in the questionnaire and their familiarity with the statements.

\subsection{Scoring Scheme}

In order to get as accurate results as possible, the questionnaire has a scoring scheme that consists of five points that represent different degrees of attitudes in order to provide the subjects with a wide range of options:1.Strongly 2. agree.3. Neutral.4.Disagree.5. Strongly disagree. See table one

Table(1) : questionnaire Communication Innovation and Text Messaging based Lingual Creativity

\begin{tabular}{|c|c|c|c|}
\hline No & Statements & $\begin{array}{l}\text { Weighted } \\
\text { Average }\end{array}$ & $\begin{array}{l}\text { Weight } \\
\text { Percentage }\end{array}$ \\
\hline 1 & $\begin{array}{l}\text { Does computer communication contribute to meet the requirements of } \\
\text { the digital era? }\end{array}$ & 78.571 & $95.3 \%$ \\
\hline 2 & $\begin{array}{l}\text { Do you think communication innovations have resulted in a variety of } \\
\text { texting requirements? }\end{array}$ & 80.357 & $100 \%$ \\
\hline 3 & $\begin{array}{l}\text { Do you think multimedia and applications have enabled us to combine } \\
\text { multimedia for the purpose of communication? }\end{array}$ & 70.238 & $86 \%$ \\
\hline 4 & Do you think that the use of acronyms is relatively small? & 56.548 & $79.1 \%$ \\
\hline 5 & $\begin{array}{l}\text { Do you think that the innovation of CMC and Twitter affected the use } \\
\text { of language? }\end{array}$ & 57.738 & $72.1 \%$ \\
\hline 6 & Do you think that the using non-standard forms puts English at risk? & 59.524 & $76.7 \%$ \\
\hline 7 & $\begin{array}{l}\text { Do you think users handling linguistics forms in the Twitter } \\
\text { communication process? }\end{array}$ & 60.119 & $83.7 \%$ \\
\hline 8 & $\begin{array}{l}\text { Do you think that the text messages are motivated by people interact } \\
\text { with this technology? }\end{array}$ & 70.833 & $95.3 \%$ \\
\hline 9 & $\begin{array}{l}\text { Do you think language innovations will contribute to the development } \\
\text { correspondence for the future? }\end{array}$ & 72.024 & $88.4 \%$ \\
\hline 10 & $\begin{array}{l}\text { Is there ignored in the personal pronouns between the interlocutor cause } \\
\text { of the language innovation? }\end{array}$ & 62.500 & $86 \%$ \\
\hline
\end{tabular}


11 Do you think delete some infinitive verbs in order to provide space that will not affect messaging innovation?

12 Are the innovation messages is stimulate people to interact and use this 80.952 technology?

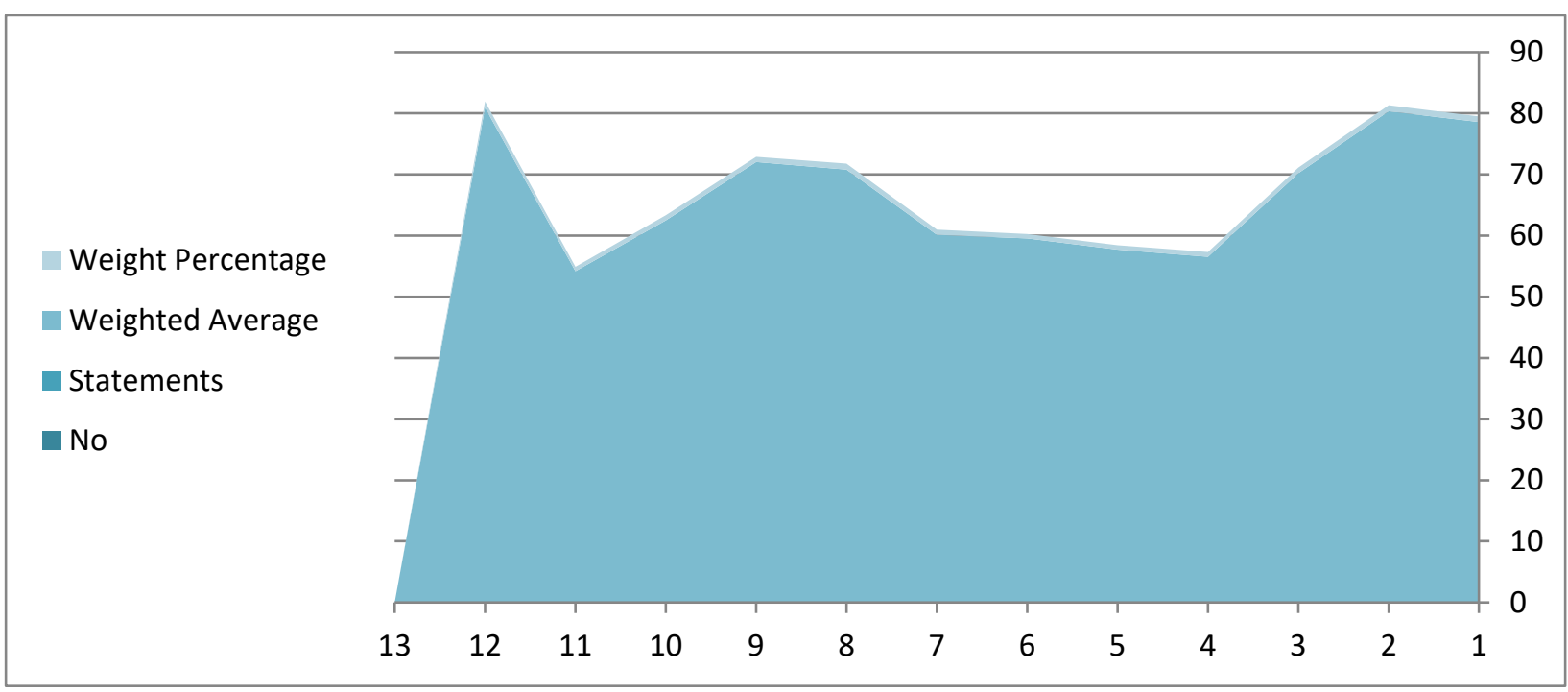

\section{The Result of The study}

The results of this study have been shown in this tables

\begin{tabular}{|l|l|l|l|l|l|}
\hline No. & Mean & Median & Std. Deviation & Variance & Percentage \% \\
\hline 1 & 1.8605 & 2.0000 & 0.86138 & 0.742 & $95.3 \%$ \\
\hline 2 & 1.7442 & 2.0000 & 0.58117 & 0.338 & $100 \%$ \\
\hline 3 & 2.2558 & 2.0000 & 1.11469 & 1.243 & $86 \%$ \\
\hline 4 & 2.7674 & 3.0000 & 0.84056 & 0.707 & $79.1 \%$ \\
\hline
\end{tabular}




\begin{tabular}{|c|c|c|c|c|c|}
\hline 5 & 2.7442 & 3.0000 & 1.13585 & 1.290 & $72.1 \%$ \\
\hline 6 & 2.6744 & 3.0000 & 1.12802 & 1.272 & $76.7 \%$ \\
\hline 7 & 2.6279 & 3.0000 & 0.81717 & 0.668 & $83.7 \%$ \\
\hline 8 & 2.2093 & 2.0000 & 0.74188 & 0.550 & $95.3 \%$ \\
\hline 9 & 2.1628 & 2.0000 & 0.92402 & 0.854 & $88.4 \%$ \\
\hline 10 & 2.4651 & 2.0000 & 0.85493 & 0.731 & $86 \%$ \\
\hline 11 & 2.8605 & 3.0000 & 0.88859 & 0.790 & $72.1 \%$ \\
\hline 12 & 1.6744 & 2.0000 & 0.60635 & 0.368 & $100 \%$ \\
\hline
\end{tabular}

Percentage \%

Variance

Std. Deviation

Median

Mean

No.

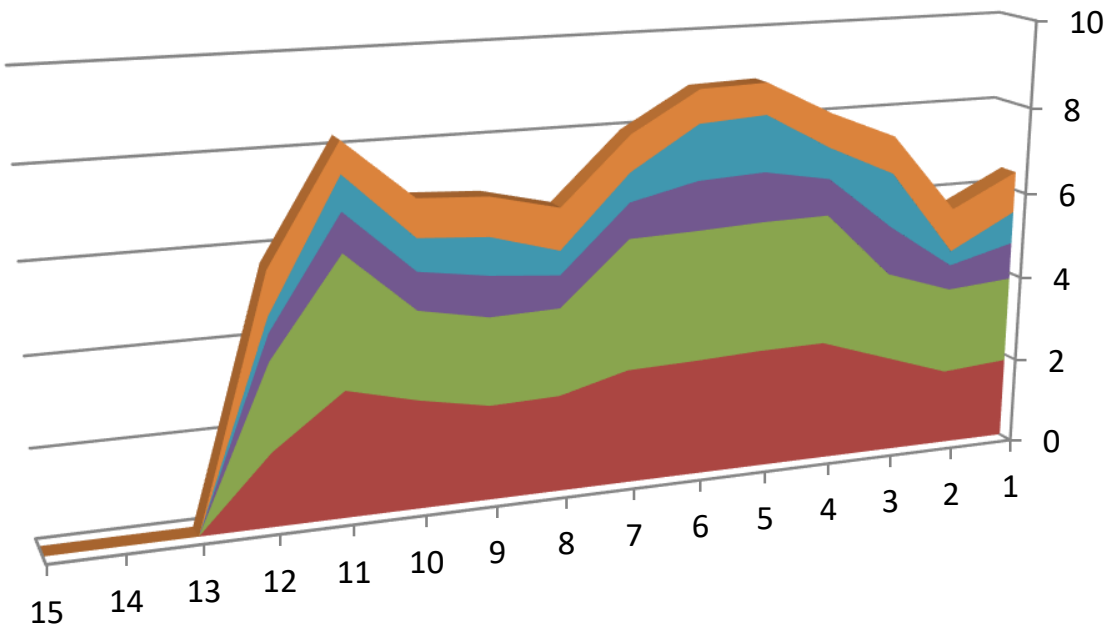




\section{CONCLUSION}

There is a belief that the use of non-standard forms online will put the future of Standard English in danger. On the other hand, it is believed "the use of shortened words and abbreviations is limited to the media. Instead, these features demonstrate the creativity of users to construct their messages, and most of the time what they want to deliver is successfully conveyed despite the non-standard forms of language that they use. Hence, rather than seeing it as degrading a language, it should be seen as users having an innovative and a good command of the language". And this innovation doesn't affect the formal language of research.

\section{REFERENCES}

Derks, D., Bos, A. E. R., \& Grumbkow, J. V. (2008). Emoticons in computer-mediated communication: Social motives and social context. CyberPsychology \& Behavior, 11(1).

Kasesniemi, E. L., \& Rautiainen, P. (2002). Mobile Culture of children and teenagers in Finland. In J. Katz \& M. Aakhus, (Eds.), Perpetual contact: Mobile Communication, Private Talk, andPublic Performance). Cambridge: Cambridge University Press.

Ling, R. (2000). We will be reached: The use of Mobile telephony among Norwegian youth. Information Technology and people.

-Ong'onda, N. A (2009). Short Message Discourse: A Sociolinguistic Approach to Kenyan text messages. Unpublished M.A. thesis, Maseno University.

-Noor Aqsa Nabila(2014) Language Use on Twitter among Malaysian L2 Speaker. Kuala Lumpur: University of Malaya.

- Tagliamonte, S. A. \& Denis, D. (2008). Linguistic ruin? LOL! Instant messaging and teen language. American Speech, $83(1)$.

-Thurlow, C., Lengel, L.B. and Tomic, A. (2004) Computer-mediated communication: social interaction and the Internet. London: Sage

-Rani Rubdy, Lubna Alsagoff (2013). the Global-Local Interface and Hybridity: 